TITLE:

\title{
Primordial gravitational waves in an inflationary braneworld
}

$\operatorname{AUTHOR}(\mathrm{S})$ :

Kobayashi, T; Kudoh, H; Tanaka, T

CITATION:

Kobayashi, T ... [et al]. Primordial gravitational waves in an inflationary braneworld. PHYSICAL REVIEW D 2003, 68(4): 044025.

ISSUE DATE:

2003-08-15

URL:

http://hdl.handle.net/2433/49919

RIGHT:

Copyright 2003 American Physical Society 
PHYSICAL REVIEW D 68, 044025 (2003)

\title{
Primordial gravitational waves in an inflationary braneworld
}

\author{
Tsutomu Kobayashi* and Hideaki Kudoh ${ }^{\dagger}$ \\ Department of Physics, Kyoto University, Kyoto 606-8502, Japan \\ Takahiro Tanaka \\ Yukawa Institute for Theoretical Physics, Kyoto University, Kyoto 606-8502, Japan
}

(Received 4 May 2003; published 28 August 2003)

\begin{abstract}
We study primordial gravitational waves from inflation in the Randall-Sundrum braneworld model. The effect of a small change of the Hubble parameter during inflation is investigated using a toy model given by connecting two de Sitter branes. We analyze the power spectrum of the final zero-mode gravitons, which is generated from the vacuum fluctuations of both the initial Kaluza-Klein modes and the zero mode. The amplitude of fluctuations is confirmed to agree with the four-dimensional one at low energies, whereas it is enhanced due to the normalization factor of the zero mode at high energies. We show that the five-dimensional spectrum can be well approximated by applying a simple mapping to the four-dimensional fluctuation amplitude.
\end{abstract}

DOI: $10.1103 /$ PhysRevD.68.044025

\section{INTRODUCTION}

Our four-dimensional universe might be embedded as a three-brane in a higher dimensional spacetime with all the standard model particles confined to the brane and with gravity allowed to propagate in the extra dimensions. This socalled braneworld scenario has opened various possibilities. A possible solution to the hierarchy problem in particle physics was presented by introducing large extra dimensions [1] or a new type of compactification [2]. Also interesting is a new possibility proposed in Ref. [3] that four-dimensional gravity is recovered effectively on the brane despite the infinite extension of the extra dimension [4,5].

The braneworld scenarios have also had a large impact on cosmology (for a review of the cosmological aspects of the braneworld scenarios, see, e.g., [6]). Homogeneous and isotropic cosmological models have been built [7], and various types of inflation models proposed [8-12]. A lot of effort has been put forth in searching for new and characteristic features of the braneworld cosmology. For example, cosmological perturbations and the related physics of the early universe have attracted great attention in the expectation that the braneworld inflation might leave their characteristic prints on the primordial spectrum of perturbations.

Although the cosmological perturbations have been discussed in a number of publications (see, e.g., [6,11-19] and references therein), the presence of the extra dimension does not allow detailed predictions about the cosmological consequences. As the simplest case, the primordial spectra of density perturbations and of gravitational (tensor) perturbations were investigated neglecting the nontrivial evolution of perturbations in the bulk in a model of slow-roll inflation driven by an inflaton field confined to the brane [12-14]. (We must mention that gravitational waves have been considered in the context of braneworld models other than the Randall-

\footnotetext{
*Electronic address: tsutomu@tap.scphys.kyoto-u.ac.jp

†Electronic address: kudoh@yukawa.kyoto-u.ac.jp

ॠElectronic address: tanaka@yukawa.kyoto-u.ac.jp
}

PACS number(s): 04.50.+h, 11.10.Kk, 98.80.Cq

Sundrum type as well; see $[20,21]$.) Although complicated, the effect on the scalar type perturbations due to the evolution of perturbations in the bulk has been discussed under some assumptions [17]. As for the gravitational wave perturbations, the authors of Ref. [18] considered a simplified inflation model in which the de Sitter stage of inflation is instantaneously connected to Minkowski space. In this model it is possible to solve the perturbation equations including the bulk to some extent. They focused on perturbations with comoving scale exceeding the Hubble scale at the end of inflation.

Also in this paper we study gravitational waves from an inflating brane. If the Hubble parameter on the brane is constant, the power spectrum becomes scale invariant [13]. However, the Hubble parameter usually changes even during inflation. The change of the Hubble parameter, i.e., the nontrivial motion of the brane in the five-dimensional bulk, "disturbs" the graviton wave function. As a result, zero-mode gravitons, which correspond to the four-dimensional gravitational waves, are created from vacuum fluctuations in the Kaluza-Klein modes as well as in the zero mode. It is also possible that gravitons initially in the zero mode escape into the extra dimension as "dark radiation." Therefore we expect that the nontrivial motion of the brane may leave characteristic features of the braneworld inflation. If so, it is interesting to search for a signature of the extra dimension left on the primordial spectrum. However, there is a technical difficulty. When the Hubble parameter is time dependent, the bulk equations are no longer separable. Then we have to solve a complicated partial differential equation. To cope with this difficulty, we consider a simple model in which two de Sitter branes are joined at a certain time; namely, we assume that the Hubble parameter changes discontinuously. In this model we can calculate the power spectrum almost analytically. This is a milder version of the transition described in [18].

This paper is organized as follows. In the next section we describe the setup of our five-dimensional model, and explain the formalism introduced in Ref. [18] to solve the mode functions for gravitational wave perturbations. Using this 
formalism, we explicitly evaluate the Bogoliubov coefficients in Sec. III. In Sec. IV we translate the results for the Bogoliubov coefficients into the power spectrum of gravitational waves, and its properties are discussed. We show that the power spectrum for our five-dimensional model can be reproduced with good accuracy from that for the corresponding four-dimensional model by applying a simple mapping. Section V is devoted to conclusion.

\section{II. de SITTER BRANE AND GRAVITATIONAL WAVE PERTURBATIONS}

\section{A. Background metric, gravitational wave perturbations, and mode functions}

Let us start with the simple case in which the background is given by a pure de Sitter brane in $\mathrm{AdS}_{5}$ bulk spacetime. We solve the five-dimensional Einstein equations for gravitational wave perturbations. For this purpose, it is convenient to use a coordinate system in which the position of the brane becomes a constant coordinate surface. In such a coordinate system the background metric is written as

$$
d s^{2}=\frac{\ell^{2}}{(\sinh \xi)^{2}}\left[\frac{1}{\eta^{2}}\left(-d \eta^{2}+\delta_{i j} d x^{i} d x^{j}\right)+d \xi^{2}\right],
$$

where $\ell$ is the bulk curvature radius, and the de Sitter brane is placed at $\xi=$ const $=\xi_{B}$. Note that here $\eta$ is supposed to be negative. On the brane, the scale factor is given by $a(\eta)=1 /(-\eta H)$ and the Hubble parameter becomes

$$
H=\ell^{-1} \sinh \xi_{B} .
$$

Note that under the coordinate transformations

$$
\begin{aligned}
& t=\eta \cosh \xi-\eta_{0} \cosh \xi_{B}, \\
& z=-\eta \sinh \xi,
\end{aligned}
$$

with a constant $\eta_{0}$, the metric (2.1) becomes the $\mathrm{AdS}_{5}$ metric in the Poincare coordinates.

The metric with gravitational wave perturbations is written as

$$
d s^{2}=\frac{\ell^{2}}{(\sinh \xi)^{2}}\left\{\frac{1}{\eta^{2}}\left[-d \eta^{2}+\left(\delta_{i j}+h_{i j}^{\mathrm{TT}}\right) d x^{i} d x^{j}\right]+d \xi^{2}\right\} .
$$

We decompose the transverse-traceless tensor $h_{i j}^{\mathrm{TT}}$ into the spatial Fourier modes as

$$
h_{i j}^{\mathrm{TT}}(\eta, \boldsymbol{x}, \xi)=\frac{\sqrt{2}}{\left(M_{5}\right)^{3 / 2}} \cdot \frac{1}{(2 \pi)^{3 / 2}} \int d^{3} p \quad \phi(\eta, \xi ; p) e^{i p \cdot x} e_{i j},
$$

where $e_{i j}$ is the polarization tensor, and the summation over different polarizations was suppressed. $M_{5}$ represents the five-dimensional Planck mass, and it is related to the fourdimensional Planck mass $M_{\mathrm{Pl}}$ by $\ell M_{5}^{3}=M_{\mathrm{Pl}}^{2}$. The factor $\sqrt{2} /\left(M_{5}\right)^{3 / 2}$ is chosen so that the effective action for $\phi$ cor- responds to the action for the canonically normalized scalar field. Then, the Einstein equations for the gravitational wave perturbations reduce to the Klein-Gordon equation for a massless scalar field in $\mathrm{AdS}_{5}$,

$$
\square \phi=\left(\mathcal{D}_{\eta}-\mathcal{D}_{\xi}\right) \phi=0,
$$

where the derivative operators are defined by

$$
\begin{aligned}
& \mathcal{D}_{\eta}=\eta^{2} \frac{\partial^{2}}{\partial \eta^{2}}-2 \eta \frac{\partial}{\partial \eta}+p^{2} \eta^{2}, \\
& \mathcal{D}_{\xi}=(\sinh \xi)^{3} \frac{\partial}{\partial \xi}(\sinh \xi)^{-3} \frac{\partial}{\partial \xi} .
\end{aligned}
$$

We assume $Z_{2}$ symmetry across the brane. Assuming that anisotropic stress is zero on the brane, Israel's junction condition gives the boundary condition for the perturbations as

$$
\left.\partial_{\xi} \phi\right|_{\xi=\xi_{B}}=0 .
$$

Since the equation is separable, the mode functions are found in the form of $\phi_{\kappa}(\eta, \xi)=\psi_{\kappa}(\eta) \chi_{\kappa}(\xi)$, where $\psi_{\kappa}(\eta)$ and $\chi_{\kappa}(\xi)$ satisfy

$$
\begin{gathered}
\left(\mathcal{D}_{\eta}+\kappa^{2}+\frac{9}{4}\right) \psi_{\kappa}(\eta)=0, \\
\left(\mathcal{D}_{\xi}+\kappa^{2}+\frac{9}{4}\right) \chi_{\kappa}(\xi)=0,
\end{gathered}
$$

respectively, and the separation constant $\kappa(\geqslant 0)$ is related to the Kaluza-Klein mass $m$ as $m^{2}=\left(\kappa^{2}+9 / 4\right) H^{2}$. For $\kappa^{2}=-9 / 4$, we have one discrete mode, which is called the zero mode. The zero-mode wave function is given by

$$
\phi_{0}^{( \pm)}=\ell^{-1 / 2} C(H) \frac{H}{\sqrt{2 p}} e^{ \pm i p \eta}\left(\eta \pm \frac{i}{p}\right),
$$

which is independent of $\xi$. The factor $C(H)$ is to be determined by the normalization condition $\left(\phi_{0}^{( \pm)} \cdot \phi_{0}^{( \pm)}\right)=\mp 1$, with respect to the Klein-Gordon inner product

$$
(F \cdot G):=-2 i \int_{\xi_{B}}^{\infty} \frac{\ell^{3} d \xi}{(\sinh \xi)^{3} \eta^{2}}\left(F \partial_{\eta} G^{*}-G^{*} \partial_{\eta} F\right) .
$$

Then we have

$$
\begin{aligned}
C^{2}(H) & =\left[2\left(\sinh \xi_{B}\right)^{2} \int_{\xi_{B}}^{\infty} d \xi \frac{1}{(\sinh \xi)^{3}}\right]^{-1} \\
& =\left[\sqrt{1+\ell^{2} H^{2}}+\ell^{2} H^{2} \ln \left(\frac{\ell H}{1+\sqrt{1+\ell^{2} H^{2}}}\right)\right]^{-1} .
\end{aligned}
$$

This normalization factor is the same that was introduced in, for example, Refs. [19,13], and behaves like 


$$
C^{2}(H) \sim\left\{\begin{array}{ccc}
1 & (\text { at low energies } & \ell H \ll 1), \\
\frac{3}{2} \ell H & (\text { at high energies } & \ell H \gg 1) .
\end{array}\right.
$$

The continuous spectrum called Kaluza-Klein (KK) modes starts with $\kappa=0$. Notice that the mode labeled by $\kappa$ $=0$ does not correspond to the zero mode [8]. Writing the positive and the negative frequency modes, respectively, as $\phi_{\kappa}^{(+)}=\psi_{\kappa}^{(+)} \chi_{\kappa}$ and $\phi_{\kappa}^{(-)}=\left(\phi_{\kappa}^{(+)}\right)^{*}=\psi_{\kappa}^{(-)} \chi_{\kappa}^{*}$, we impose the conditions

$$
\begin{gathered}
\frac{i \ell^{3}}{\eta^{2}}\left(\psi_{\kappa}^{(+)} \partial_{\eta} \psi_{\kappa}^{(-)}-\text {c.c. }\right)=1, \\
2 \int_{\xi_{B}}^{\infty} \frac{d \xi}{(\sinh \xi)^{3}} \chi_{\kappa^{\prime}}^{*} \chi_{\kappa}=\delta\left(\kappa-\kappa^{\prime}\right),
\end{gathered}
$$

So as to satisfy the normalization condition $\left(\phi_{\kappa}^{( \pm)} \cdot \phi_{\kappa^{\prime}}^{( \pm)}\right)$ $=\mp \delta\left(\kappa-\kappa^{\prime}\right)$. The solutions of Eq. (2.9) are given in terms of the Hankel functions by

$$
\psi_{\kappa}^{(-)}(\eta)=\frac{\sqrt{\pi}}{2} e^{-3 / 2} e^{-\pi \kappa / 2}|\eta|^{3 / 2} H_{i \kappa}^{(1)}(p|\eta|) .
$$

The spatial mode function $\chi_{\kappa}(\xi)$ is given in terms of the associated Legendre functions by $[11]$

$$
\chi_{\kappa}=C_{1}(\sinh \xi)^{2}\left[P_{-1 / 2+i \kappa}^{-2}(\cosh \xi)-C_{2} Q_{-1 / 2+i \kappa}^{-2}(\cosh \xi)\right],
$$

where from Eqs. (2.8) and (2.16) the constants $C_{1}$ and $C_{2}$ are

$$
\begin{aligned}
C_{1}= & {\left[\left|\frac{\Gamma(i \kappa)}{\Gamma(5 / 2+i \kappa)}\right|^{2}\right.} \\
& \left.+\left|\frac{\Gamma(-i \kappa)}{\Gamma(5 / 2-i \kappa)}-\pi C_{2} \frac{\Gamma(i \kappa-3 / 2)}{\Gamma(1+i \kappa)}\right|^{2}\right]^{-1 / 2}, \\
C_{2}= & \frac{P_{-1 / 2+i \kappa}^{-1}\left(\cosh \xi_{B}\right)}{Q_{-1 / 2+i \kappa}^{-1}\left(\cosh \xi_{B}\right)} .
\end{aligned}
$$

As will be seen, we need to evaluate the value of the wave function at the location of the brane $\chi_{\kappa}\left(\xi_{B}\right)$, and in some special cases $\chi_{\kappa}\left(\xi_{B}\right)$ reduces to a rather simple form. For $\sinh \xi_{B} \ll 1$ and $\kappa \sinh \xi_{B} \ll 1$, we have

$$
\chi_{\kappa}\left(\xi_{B}\right) \approx \sqrt{\frac{\kappa \tanh \pi \kappa}{2}} \sqrt{\frac{\kappa^{2}+1 / 4}{\kappa^{2}+9 / 4}}\left(\sinh \xi_{B}\right)^{2},
$$

while, for $\sinh \xi_{B} \gg 1$ or $\kappa \sinh \xi_{B} \gg 1$, we have

$$
\chi_{\kappa}\left(\xi_{B}\right) \approx \frac{1}{\sqrt{\pi}}\left(\sinh \xi_{B}\right)^{3 / 2} \frac{\kappa}{\sqrt{\kappa^{2}+9 / 4}} .
$$

For the derivation of these two expressions, see Ref. [18].

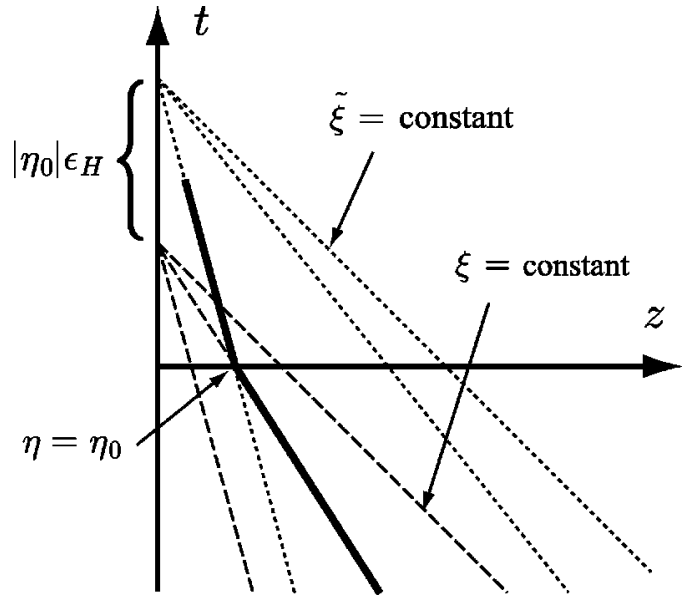

FIG. 1. Trajectory of the brane (thick solid line) in static coordinates. Dashed (dotted) lines represent surfaces of $\xi=$ const $(\widetilde{\xi}$ $=$ const $)$.

\section{B. Model with a jump in the Hubble parameter}

We consider a model in which the Hubble parameter changes during inflation. As we have explained, in the case of constant Hubble parameter the brane can be placed at a constant coordinate plane. When the Hubble parameter varies, we need to consider a moving brane in the same coordinates. For simplicity, we consider the situation in which the Hubble parameter changes discontinuously at $\eta=\eta_{0}$ from $H_{1}$ to

$$
H_{2}=H_{1}-\delta H \text {. }
$$

Here $\delta H / H_{1}$ is assumed to be small. For later convenience, we define a small quantity $\epsilon_{H}$ by

$$
\begin{aligned}
\epsilon_{H}= & \frac{\ell H_{1} \sqrt{1+\left(\ell H_{2}\right)^{2}}-\ell H_{2} \sqrt{1+\left(\ell H_{1}\right)^{2}}}{\ell H_{2}} \\
= & \frac{1}{\sqrt{1+\left(\ell H_{1}\right)^{2}}} \frac{\delta H}{H_{1}}+\frac{2+3\left(\ell H_{1}\right)^{2}}{2\left(1+\left(\ell H_{1}\right)^{2}\right)^{3 / 2}}\left(\frac{\delta H}{H_{1}}\right)^{2} \\
& +\mathcal{O}\left(\frac{\delta H}{H_{1}}\right)^{3} .
\end{aligned}
$$

To describe the motion of the de Sitter brane after transition, it is natural to introduce a new coordinate system $(\tilde{\eta}, \widetilde{\xi})$ defined by

$$
\begin{aligned}
& t=\tilde{\eta} \cosh \tilde{\xi}-\tilde{\eta}_{0} \cosh \widetilde{\xi}_{B}, \\
& z=-\tilde{\eta} \sinh \tilde{\xi} .
\end{aligned}
$$

Then, the brane expanding with Hubble parameter $H_{2}$ is placed at $\widetilde{\xi}=\widetilde{\xi}_{B}$ by choosing two constants $\widetilde{\xi}_{B}$ and $\widetilde{\eta}_{0}$ so as to satisfy $H_{2}=\ell^{-1} \sinh \widetilde{\xi}_{B}$ and $\eta_{0} \sinh \xi_{B}=\widetilde{\eta}_{0} \sinh \widetilde{\xi}_{B}$. The trajectory of the brane is shown in Fig. 1. Apparently, mode functions in this coordinate system take the same form as those in the previous section, but the arguments $(\xi, \eta)$ and 
the Hubble parameter $H_{1}$ are replaced by $(\tilde{\xi}, \tilde{\eta})$ and $H_{2}$. We refer to these second set of modes as $\widetilde{\phi}_{0}$ and $\widetilde{\phi}_{\kappa}$. The relation between $(\eta, \xi)$ and $(\tilde{\eta}, \widetilde{\xi})$ is

$$
\begin{aligned}
\tilde{\eta} & =-\sqrt{\eta^{2}+2 \epsilon_{H} \eta_{0} \eta \cosh \xi+\epsilon_{H}^{2} \eta_{0}^{2}}, \\
\tanh \tilde{\xi} & =\left(\eta \cosh \xi+\epsilon_{H} \eta_{0}\right)^{-1} \eta \sinh \xi .
\end{aligned}
$$

As explained above, the variation of the Hubble parameter is assumed to be small. For a technical reason, we further impose a weak restriction that the wavelength of the perturbations concerned is larger than $\delta H / H^{2}$. These conditions are summarized as follows:

$$
\begin{array}{r}
\frac{\delta H}{H} \ll 1, \\
p\left|\eta_{0}\right| \frac{\delta H}{H} \ll 1 .
\end{array}
$$

\section{Method to calculate Bogoliubov coefficients}

We consider the graviton wave function $\varphi$ that becomes the zero mode $\widetilde{\phi}_{0}^{(-)}$at the infinite future $\tilde{\eta}=0$. We write the wave function $\varphi$ as

$$
\varphi=\widetilde{\phi}_{0}^{(-)}+\delta \varphi
$$

where the second term $\delta \varphi$ arises because $\widetilde{\phi}_{0}^{(-)}$does not satisfy the boundary condition for $t<0$. Writing down $\widetilde{\phi}_{0}^{(-)}$and $\delta \varphi$ at the infinite past, $\eta=-\infty$, as a linear combination of $\phi_{0}^{( \pm)}$and $\phi_{\kappa}^{( \pm)}$, we can read the Bogoliubov coefficients.

At $\eta \rightarrow-\infty$ the first term $\widetilde{\phi}_{0}^{(-)}$is expanded as

$$
\widetilde{\phi}_{0}^{(-)} \underset{\eta \rightarrow-\infty}{\rightarrow} \sum_{M=0, \kappa}\left(U_{0 M} \phi_{M}^{(-)}+V_{0 M} \phi_{M}^{(+)}\right)
$$

where the summation is taken over the zero mode and the KK modes. The coefficients $U_{0 \kappa}$ and $V_{0 \kappa}$ are written by using the inner product as

$$
\begin{aligned}
& U_{0 M}=\lim _{\eta \rightarrow-\infty}\left(\widetilde{\phi}_{0}^{(-)} \cdot \phi_{M}^{(-)}\right), \\
& V_{0 M}=-\lim _{\eta \rightarrow-\infty}\left(\widetilde{\phi}_{0}^{(-)} \cdot \phi_{M}^{(+)}\right) .
\end{aligned}
$$

Evaluation of the inner product at $\eta=-\infty$ leads to $V_{00}$ $=V_{0 \kappa}=0[18]$, while

$$
\begin{aligned}
U_{00} \approx & \frac{H_{2} C\left(H_{2}\right)}{H_{1} C\left(H_{1}\right)} e^{-i \epsilon_{H} p \eta_{0} \cosh \xi_{B}\left[1-i \epsilon_{H} p \eta_{0}\left[C^{2}\left(H_{1}\right)\right.\right.} \\
& \left.\left.-\cosh \xi_{B}\right]-\frac{1}{2}\left(\epsilon_{H} p \eta_{0}\right)^{2}\left(\sinh \xi_{B}\right)^{2}\right],
\end{aligned}
$$

$$
U_{0 \kappa} \approx \epsilon_{H} p \eta_{0} \frac{-2 i e^{i \pi / 4}}{\kappa^{2}+1 / 4} \frac{\ell H_{2}}{\left(\ell H_{1}\right)^{2}} C\left(H_{2}\right) \chi_{\kappa}\left(\xi_{B}\right) \text {. }
$$

These expressions are approximately correct as long as $p\left|\eta_{0}\right| \delta H / H_{1} \ll 1$ is satisfied. The derivation of these equations is explained in Appendix B.

The second term $\delta \varphi$ in Eq. (2.28) is obtained as follows. Since both $\varphi$ and $\widetilde{\phi}_{0}^{(-)}$satisfy the Klein-Gordon equation in the bulk (2.6), $\delta \varphi$ also obeys the same equation. The boundary condition for $\delta \varphi$ is derived from Eq. (2.8) as $\partial_{\xi} \delta \varphi$ $=-\partial_{\xi} \widetilde{\phi}_{0}^{(-)}$. Therefore, the solution $\delta \varphi$ is given by

$$
\delta \varphi=2 \int_{-\infty}^{\eta_{0}} d \eta^{\prime} G_{\mathrm{adv}}\left(\eta, \xi ; \eta^{\prime}, \xi_{B}\right)\left[\partial_{\xi^{\prime}} \widetilde{\phi}_{0}^{(-)}\left(\eta^{\prime}, \xi^{\prime}\right)\right]_{\xi^{\prime}=\xi_{B}}
$$

with the aid of the advanced Green's function that satisfies

$$
\left(\mathcal{D}_{\eta}-\mathcal{D}_{\xi}\right) G_{\mathrm{adv}}\left(\eta, \xi ; \eta^{\prime}, \xi^{\prime}\right)=\delta\left(\eta-\eta^{\prime}\right) \delta\left(\xi-\xi^{\prime}\right)
$$

The explicit form of the Green's function is [18]

$$
\begin{aligned}
G_{\mathrm{adv}}\left(\eta, \xi ; \eta^{\prime}, \xi^{\prime}\right)= & \sum_{M} \frac{i \ell^{3}}{\left(\sinh \xi^{\prime}\right)^{3} \eta^{\prime 4}} \theta\left(\eta^{\prime}-\eta\right) \\
& \times\left[\phi_{M}^{(+)}(\eta, \xi) \phi_{M}^{(-)}\left(\eta^{\prime}, \xi^{\prime}\right)\right. \\
& \left.-\phi_{M}^{(-)}(\eta, \xi) \phi_{M}^{(+)}\left(\eta^{\prime}, \xi^{\prime}\right)\right]
\end{aligned}
$$

Taking the limit $\eta \rightarrow-\infty$, we can expand $\delta \varphi$ in terms of in-vacuum mode functions,

$$
\delta \varphi \underset{\eta \rightarrow-\infty}{\rightarrow} \sum_{M=0, \kappa}\left[u_{0 M} \phi_{M}^{(-)}+v_{0 M} \phi_{M}^{(+)}\right]
$$

where the coefficients are given by

$$
\begin{aligned}
u_{0 M}= & -2 i \ell^{3} \int_{-\infty}^{\eta_{0}} \frac{d \eta}{\left(\sinh \xi_{B}\right)^{3} \eta^{4}} \phi_{M}^{(+)}\left(\eta, \xi_{B}\right) \\
& \times\left[\partial_{\xi} \widetilde{\phi}_{0}^{(-)}(\eta, \xi)\right]_{\xi=\xi_{B}}, \\
v_{0 M}= & 2 i \ell^{3} \int_{-\infty}^{\eta_{0}} \frac{d \eta}{\left(\sinh \xi_{B}\right)^{3} \eta^{4}} \phi_{M}^{(-)}\left(\eta, \xi_{B}\right) \\
& \times\left[\partial_{\xi} \widetilde{\phi}_{0}^{(-)}(\eta, \xi)\right]_{\xi=\xi_{B}} .
\end{aligned}
$$

To evaluate these coefficients, we need the source term $\left.\partial_{\xi} \widetilde{\phi}_{0}^{(-)}\right|_{\xi=\xi_{B}}$ written in terms of the coordinates $(\eta, \xi)$, which is

$$
\begin{aligned}
\left.\partial_{\xi} \widetilde{\phi}_{0}^{(-)}\right|_{\xi=\xi_{B}}= & -\ell^{-3 / 2} \sinh \underset{\xi_{B} \frac{C\left(H_{2}\right)}{\sqrt{2 p}}}{ } \\
& \times i p \epsilon_{H} \eta_{0} \eta \sinh \xi_{B} e^{i p \sqrt{\eta^{2}+2 \epsilon_{H} \eta_{0} \eta \cosh \xi_{B}+\epsilon_{H}^{2} \eta_{0}^{2}}} .
\end{aligned}
$$


From Eqs. (2.29) and (2.36), we finally obtain the Bogoliubov coefficients relating the initial zero mode or KK modes to the final zero mode,

$$
\varphi=\sum_{M}\left(\alpha_{0 M} \phi_{M}^{(-)}+\beta_{0 M} \phi_{M}^{(+)}\right)
$$

where

$$
\begin{aligned}
& \alpha_{0 M}=U_{0 M}+u_{0 M}, \\
& \beta_{0 M}=v_{0 M} .
\end{aligned}
$$

From these coefficients we can evaluate the number and the power spectrum of the generated gravitons.

\section{EVALUATION OF BOGOLIUBOV COEFFICIENTS}

Now let us evaluate the expressions for the Bogoliubov coefficients obtained in the preceding section. We concentrate on the two limiting cases: the low energy regime $\left(\ell H_{1} \ll 1\right)$ and high energy regime $\left(\ell H_{1} \gg 1\right)$. We first evaluate the coefficients $\alpha_{00}$ and $\beta_{00}$, which relate the initial zero mode to the final zero mode. We keep the terms up to second order in $\epsilon_{H}$ (or equivalently in $\delta H / H_{1}$ ).

Substituting Eq. (2.39) into Eq. (2.38), we have

$$
\begin{aligned}
\beta_{00}= & C\left(H_{1}\right) C\left(H_{2}\right) \frac{H_{2}}{H_{1}} \epsilon_{H} \eta_{0} \int_{-\infty}^{\eta_{0}} d \eta\left(\frac{1}{\eta^{2}}-\frac{i}{p \eta^{3}}\right) \\
& \times e^{-i p\left(\eta-\sqrt{\eta^{2}+2 \epsilon_{H} \eta_{0} \eta \cosh \xi_{B}+\epsilon_{H}^{2} \eta_{0}^{2}}\right)} .
\end{aligned}
$$

Because there is a factor $\epsilon_{H}$ in front of the integral, we can neglect the correction of $O\left(\epsilon_{H}^{2}\right)$ in the integrand. Then we can carry out the integration to obtain

$$
\beta_{00} \approx C\left(H_{1}\right) C\left(H_{2}\right) \frac{H_{2}}{H_{1}} \epsilon_{H} \frac{i}{2 p \eta_{0}} e^{-2 i p \eta_{0}-i p \eta_{0} \epsilon_{H} \cosh \xi_{B}} .
$$

Similarly, we get

$$
\alpha_{00} \approx U_{00}+C\left(H_{1}\right) C\left(H_{2}\right) \frac{H_{2}}{H_{1}} \epsilon_{H}\left(1+\frac{i}{2 p \eta_{0}}\right) e^{-i p \eta_{0} \epsilon_{H} \cosh \xi_{B}},
$$

where $U_{00}$ is given by Eq. (2.31).

At low energies $\left(\ell H_{1} \ll 1\right)$, the Bogoliubov coefficients $\alpha_{00}$ and $\beta_{00}$ become

$$
\begin{aligned}
& \alpha_{00} \approx\left[1+\frac{i}{2 p \eta_{0}} \frac{\delta H}{H_{1}}\right] e^{-i p \eta_{0} \delta H / H_{2}}, \\
& \beta_{00} \approx \frac{i}{2 p \eta_{0}} \frac{\delta H}{H_{1}} e^{-2 i p \eta_{0}-i p \eta_{0} \delta H / H_{2}} .
\end{aligned}
$$

It is worth noting that these expressions are correct up to second order in $\delta H / H_{1}$. This result agrees with the results of the four-dimensional calculation, $\alpha^{(4 \mathrm{D})}$ and $\beta^{(4 \mathrm{D})}$.

At high energies $\left(\ell H_{1} \gg 1\right)$, the coefficients are

$$
\begin{aligned}
\alpha_{00} \approx & {\left[1+\frac{3}{2}\left(\frac{i}{2 p \eta_{0}} \frac{\delta H}{H_{1}}\right)+\frac{3}{8}\left(\frac{\delta H}{H_{1}}\right)^{2}-\frac{i p \eta_{0}}{2} \frac{\delta H}{H_{1}}\right.} \\
& \left.-\frac{\left(p \eta_{0}\right)^{2}}{2}\left(\frac{\delta H}{H_{1}}\right)^{2}\right] e^{-i p \eta_{0} \delta H / H_{1}-i p \eta_{0}(3 / 2)\left(\delta H / H_{1}\right)^{2}},
\end{aligned}
$$

$$
\beta_{00} \approx \frac{3}{2}\left(\frac{i}{2 p \eta_{0}} \frac{\delta H}{H_{1}}\right) e^{-2 i p \eta_{0}-i p \eta_{0} \delta H / H_{1}-i p \eta_{0}(3 / 2)\left(\delta H / H_{1}\right)^{2}} .
$$

Here we stress that the last two terms in the square brackets of Eq. (3.6), both coming from $U_{00}$, are enhanced at $p\left|\eta_{0}\right|$ $\gg 1$.

Next, we calculate the Bogoliubov coefficients $\alpha_{0 \kappa}$ and $\beta_{0 \kappa}$, which relate the initial $\mathrm{KK}$ modes to the final zero mode, up to the leading first order in $\delta H / H_{1}$. Although we will calculate the power spectrum up to second order in $\delta H / H_{1}$ in the next section, the expressions up to first order are sufficient for $\alpha_{0 \kappa}$ and $\beta_{0 \kappa}$, in contrast to the case for $\alpha_{00}$ and $\beta_{00}$. Again, substituting $\partial_{\xi} \widetilde{\phi}_{0}^{(-)}\left(\eta, \xi_{B}\right)$ into Eq. (2.38), we have

$$
\begin{aligned}
\beta_{0 \kappa}= & \sqrt{2 p} \ell^{3 / 2} C\left(H_{2}\right) \chi_{\kappa}^{*}\left(\xi_{B}\right) \frac{\sinh \widetilde{\xi}_{B}}{\left(\sinh \xi_{B}\right)^{2}} \\
& \times \epsilon_{H} \eta_{0} \int_{-\infty}^{\eta_{0}} \frac{d \eta}{\eta^{3}} \psi_{\kappa}^{(-)}(\eta) e^{i p \sqrt{\eta^{2}+2 \epsilon_{H} \eta_{0} \eta \cosh \xi_{B}+\epsilon_{H}^{2} \eta_{0}^{2}}} .
\end{aligned}
$$

Setting $\epsilon_{H}$ in the integrand to zero, the coefficient reduces to

$$
\begin{aligned}
\beta_{0 \kappa} \approx & \sqrt{\frac{\pi}{2}} C\left(H_{2}\right) \chi_{\kappa}^{*}\left(\xi_{B}\right) \frac{\ell H_{2}}{\left(\ell H_{1}\right)^{2}} \\
& \times \epsilon_{H} p \eta_{0} e^{-\pi \kappa / 2} \int_{\infty}^{p\left|\eta_{0}\right|} d x x^{-3 / 2} H_{i \kappa}^{(1)}(x) e^{i x},
\end{aligned}
$$

where we have introduced the integration variable $x=-p \eta$. Similarly, we have

$$
\begin{aligned}
\alpha_{0 \kappa}^{*} \approx & U_{0 \kappa}^{*}-\sqrt{\frac{\pi}{2}} C\left(H_{2}\right) \chi_{\kappa}^{*}\left(\xi_{B}\right) \frac{\ell H_{2}}{\left(\ell H_{1}\right)^{2}} \\
& \times \epsilon_{H} p \eta_{0} e^{-\pi \kappa / 2} \int_{\infty}^{p\left|\eta_{0}\right|} d x x^{-3 / 2} H_{i \kappa}^{(1)}(x) e^{-i x},
\end{aligned}
$$

where $U_{0 \kappa}$ is given by Eq. (2.32) and is $\mathcal{O}\left(\epsilon_{H}\right){ }^{1}$

Now let us discuss the dependence of $\alpha_{0 \kappa}$ and $\beta_{0 \kappa}$ on $\ell H_{1}$ and $\delta H / H_{1}$ in the limiting cases $\ell H_{1} \ll 1$ and $\ell H_{1}$ $\gg 1$. At low energies, we find, using Eq. (2.20),

\footnotetext{
${ }^{1}$ The integral including the Hankel function is written in terms of generalized hypergeometric functions.
} 


$$
\left|\beta_{0 \kappa}\right|^{2},\left|\alpha_{0 \kappa}\right|^{2} \propto\left(\ell H_{1}\right)^{2}\left(\frac{\delta H}{H_{1}}\right)^{2} \quad\left(\ell H_{1} \ll 1\right),
$$

where we have omitted the dependence on $\kappa$ and $p\left|\eta_{0}\right|$. These coefficients are suppressed by the factors of $\ell H_{1}$ and $\delta H / H_{1}$. Recall that $\alpha_{00}$ and $\beta_{00}$ agree with the standard four-dimensional result at low energies. Thus, because of the suppression of $\alpha_{0 \kappa}$ and $\beta_{0 \kappa}$ at low energies, the fourdimensional result is recovered only by the contribution from the initial zero mode.

At high energies, we obtain from Eq. (2.21)

$$
\left|\beta_{0 \kappa}\right|^{2},\left|\alpha_{0 \kappa}\right|^{2} \propto\left(\frac{\delta H}{H_{1}}\right)^{2} \quad\left(\ell H_{1} \gg 1\right),
$$

where we have again omitted the dependence on $\kappa$ and $p\left|\eta_{0}\right|$. In contrast to the result in the low energy regime (3.11), this high energy behavior is not associated with any suppression factor.

Integrating $\left|\beta_{0 \kappa}\right|^{2}$ over the KK continuum, we obtain the total number of zero-mode gravitons created from the initial KK vacuum. It can be shown that the coefficients behave as $\beta_{0 \kappa} \approx-\alpha_{0 \kappa}^{*} \sim\left(p\left|\eta_{0}\right|\right)^{1 / 2}$ at $p\left|\eta_{0}\right| \ll 1$, and we have

$$
\beta_{0 \kappa}+\alpha_{0 \kappa}^{*} \sim \mathcal{O}\left(p\left|\eta_{0}\right|\right)^{3 / 2} .
$$

Thus the number of gravitons created is proportional to $p$ outside the horizon and is evaluated as

$$
\int_{0}^{\infty}\left|\beta_{0 \kappa}\right|^{2} d \kappa \approx\left\{\begin{array}{cc}
0.5 \times p\left|\eta_{0}\right|\left(\ell H_{1}\right)^{2}\left(\frac{\delta H}{H_{1}}\right)^{2} & \left(\ell H_{1} \ll 1\right), \\
0.3 \times p\left|\eta_{0}\right|\left(\frac{\delta H}{H_{1}}\right)^{2} & \left(\ell H_{1} \gg 1\right) .
\end{array}\right.
$$

On the other hand, making use of the asymptotic form of the Hankel function $H_{\nu}^{(1)}(x) \sim e^{i(x-(2 \nu+1) \pi / 4)} / \sqrt{x}$ for $x \rightarrow \infty$, we can evaluate the integral in Eq. (3.9) in the $p\left|\eta_{0}\right| \rightarrow \infty$ limit as

$$
\beta_{0 \kappa} \propto p\left|\eta_{0}\right| \int_{\infty}^{p\left|\eta_{0}\right|} d x \frac{e^{2 i x}}{x^{2}} \sim \frac{1}{p\left|\eta_{0}\right|}\left(p\left|\eta_{0}\right| \rightarrow \infty\right),
$$

where we have carried out the integration by parts and kept the most dominant term. This shows that the creation of gravitons is suppressed well inside the horizon. Since the assumption of the instantaneous transition tends to overestimate particle production at large $p$ [22], the number of particles created from the initial zero mode and KK modes is expected to be more suppressed inside the horizon than Eq. (3.15) if we consider a realistic situation in which the Hubble parameter changes smoothly. Note that $u_{0 \kappa}$ $\propto p\left|\eta_{0}\right| \int_{\infty}^{p\left|\eta_{0}\right|} x^{-2} d x$ is constant for $p\left|\eta_{0}\right| \rightarrow \infty$. Therefore, the $\alpha_{0 \kappa}$ coefficient is dominated by $U_{0 \kappa}$ at large $p$, which behaves like $\left|\alpha_{0 \kappa}\right|^{2} \sim\left|U_{0 \kappa}\right|^{2} \propto\left(p \eta_{0}\right)^{2}$.

An example of numerical calculation is shown in Fig. 2. The figure shows that the spectrum has a peak around the

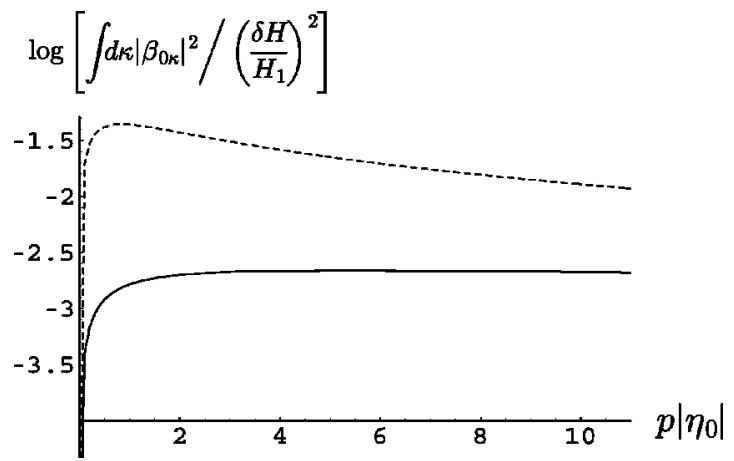

FIG. 2. Spectra of number density of zero-mode gravitons created from vacuum fluctuations in the initial KK modes. Integration over $\kappa$ is performed numerically. The solid line represents the low energy case with $\ell H_{1}=0.1$, while the dashed line shows the high energy case. The latter should be understood as the limiting case $\ell H_{1} \rightarrow \infty$ since at high energies the number does not depend on $\ell H_{1}$; see Eq. (3.12).

Hubble scale and then decreases inside the horizon, and we confirmed that the behavior of the number density outside the horizon is well described by Eq. (3.14).

\section{POWER SPECTRUM OF GENERATED GRAVITATIONAL WAVES}

So far, we have discussed the Bogoliubov coefficients to see the number of created gravitons. However, our main interest is in the power spectrum of gravitational waves because the meaning of "particle" is obscure at the super Hubble scale.

\section{A. Pure de Sitter brane}

Gravitational waves generated from pure de Sitter inflation on a brane have the scale invariant spectrum

$$
\mathcal{P}_{5 \mathrm{D}}=\frac{2 C^{2}(H)}{M_{\mathrm{Pl}}^{2}}\left(\frac{H}{2 \pi}\right)^{2},
$$

which is defined by the expectation value of the squared amplitude of the vacuum fluctuation, $8 \pi p^{3}\left|\phi_{0}\right|^{2} /\left(2 \pi M_{5}\right)^{3}$, evaluated at a late time; see Eq. (2.11). Since $C^{2} \sim 1$ at $\ell H$ $\ll 1$, this power spectrum agrees at low energies with the standard four-dimensional spectrum

$$
\mathcal{P}_{4 \mathrm{D}}=\frac{2}{M_{\mathrm{Pl}}^{2}}\left(\frac{H}{2 \pi}\right)^{2} .
$$

At high energies, however, the power spectrum (4.1) is enhanced due to the factor $C(H)$, and is much greater than the four-dimensional counterpart. This amplification effect was found in Ref. [13]. These results say that the difference between Eq. (4.1) and Eq. (4.2) is absorbed by the transformation

$$
H \mapsto H C(H) .
$$




\section{B. Model with variation of Hubble parameter}

Now let us turn to the case in which the Hubble parameter is not constant. Time variation of the Hubble parameter during inflation brings a small modification to the spectrum, and the resulting spectrum depends on the wavelength. Here we consider the amplitude of vacuum fluctuation of the zero mode in the final state. It will be a relevant observable for the observers on the brane at a late epoch because the KK mode fluctuations at super Hubble scale rapidly decay in the expanding universe due to its four-dimensional effective mass. Since the behavior of the zero-mode wave function at the infinite future $\tilde{\eta} \rightarrow 0$ is known from Eq. (2.11), we find that the vacuum fluctuation for zero mode at a late epoch is given by

$$
\begin{gathered}
\lim _{\tilde{\eta} \rightarrow 0}\left|\alpha_{00} \widetilde{\phi}_{0}^{(+)}-\beta_{00}^{*} \widetilde{\phi}_{0}^{(-)}\right|^{2}+\int d \kappa\left|\alpha_{0 \kappa} \widetilde{\phi}_{0}^{(+)}-\beta_{0 \kappa}^{*} \widetilde{\phi}_{0}^{(-)}\right|^{2} \\
=\frac{C^{2}\left(H_{2}\right)}{\ell} \frac{H_{2}^{2}}{2 p^{3}}\left(\left|\alpha_{00}+\beta_{00}^{*}\right|^{2}+\int d \kappa\left|\alpha_{0 \kappa}+\beta_{0 \kappa}^{*}\right|^{2}\right) .
\end{gathered}
$$

Multiplying this by $\left(2 / M_{5}^{3}\right)\left(p^{3} / 2 \pi^{2}\right)$ and recalling the relation $\ell M_{5}^{3}=M_{\mathrm{Pl}}^{2}$, we obtain the power spectrum

$$
\mathcal{P}_{5 \mathrm{D}}(p)=\mathcal{P}_{5 \mathrm{D}}^{\mathrm{zero}}(p)+\mathcal{P}_{5 \mathrm{D}}^{\mathrm{KK}}(p),
$$

with

$$
\begin{aligned}
& \mathcal{P}_{5 \mathrm{D}}^{\mathrm{zero}}(p)=\frac{2 C^{2}\left(H_{2}\right)}{M_{\mathrm{Pl}}^{2}}\left(\frac{H_{2}}{2 \pi}\right)^{2}\left|\alpha_{00}+\beta_{00}^{*}\right|^{2}, \\
& \mathcal{P}_{5 \mathrm{D}}^{\mathrm{KK}}(p)=\frac{2 C^{2}\left(H_{2}\right)}{M_{\mathrm{Pl}}^{2}}\left(\frac{H_{2}}{2 \pi}\right)^{2} \int_{0}^{\infty} d \kappa\left|\alpha_{0 \kappa}+\beta_{0 \kappa}^{*}\right|^{2} .
\end{aligned}
$$

The power spectrum in the four-dimensional theory, computed in the same way, is given by

$$
\mathcal{P}_{4 \mathrm{D}}(p)=\frac{2}{M_{\mathrm{Pl}}^{2}}\left(\frac{H_{2}}{2 \pi}\right)^{2}\left|\alpha^{(4 \mathrm{D})}+\beta^{*(4 \mathrm{D})}\right|^{2} \text {. }
$$

The appearance of the coefficient $\alpha$ in the power spectrum may look unusual. This is due to our setup, in which the final state of the universe is still inflating. In such a case, the fluctuations that have left the Hubble horizon never reenter it. Outside the horizon, the number of particles created, does not correspond to the power spectrum.

There are two apparent differences between $\mathcal{P}_{5 \mathrm{D}}$ and $\mathcal{P}_{4 \mathrm{D}}$; the normalization factor $C(H)$ and the contribution from the $\mathrm{KK}$ modes $\mathcal{P}_{5 \mathrm{D}}^{\mathrm{KK}}$. In the low energy regime, however, the two spectra agree with each other:

$$
\mathcal{P}_{5 \mathrm{D}} \approx \mathcal{P}_{4 \mathrm{D}}\left(\ell H_{1} \ll 1\right) \text {. }
$$

This is because, as seen from the discussion about the Bogoliubov coefficients in the preceding section, the zero-mode contribution $\mathcal{P}_{5 \mathrm{D}}^{\text {zero }}$ is exactly the same as $\mathcal{P}_{4 \mathrm{D}}$ (up to the nor- malization factor), and the Kaluza-Klein contribution $\mathcal{P}_{5 \mathrm{D}}^{\mathrm{KK}}$ is suppressed by the factor $\left(\ell H_{1}\right)^{2}$. On the other hand, when $\ell H_{1}$ is large, the amplitude of gravitational waves deviates from the four-dimensional one owing to the amplification of the factor $C(H)$.

We have observed for the pure de Sitter inflation that the correspondence between the five-dimensional power spectrum and the four-dimensional one is realized by the map (4.3). It is interesting to investigate whether the correspondence can be generalized to the present case. It seems natural to give the transformation in this case by

$$
h \mapsto h C(h),
$$

where

$$
h(p)=H_{2}\left|\alpha^{(4 \mathrm{D})}+\beta^{*(4 \mathrm{D})}\right|
$$

namely, the rescaled power spectrum $\mathcal{P}_{\text {res }}(p)$ is defined as

$$
\mathcal{P}_{\text {res }}(p)=\frac{2 C^{2}(h)}{M_{\mathrm{Pl}}^{2}}\left(\frac{h}{2 \pi}\right)^{2} .
$$

We will see that this transformation works well and mostly absorbs the difference between the five- and fourdimensional amplitudes.

We examine the differences between the five-dimensional spectrum and the rescaled four-dimensional spectrum by expanding them with respect to $\delta H / H_{1}$ as

$$
\begin{aligned}
& \mathcal{P}_{5 \mathrm{D}}(p)=\mathcal{P}_{5 \mathrm{D}}^{(0)}+\mathcal{P}_{5 \mathrm{D}}^{(1)}+\mathcal{P}_{5 \mathrm{D}}^{(2)}+\mathcal{O}\left(\frac{\delta H}{H}\right)^{3}+\mathcal{P}_{5 \mathrm{D}}^{\mathrm{KK}}, \\
& \mathcal{P}_{\text {res }}(p)=\mathcal{P}_{\text {res }}^{(0)}+\mathcal{P}_{\text {res }}^{(1)}+\mathcal{P}_{\text {res }}^{(2)}+\mathcal{O}\left(\frac{\delta H}{H}\right)^{3} .
\end{aligned}
$$

Here the quantity associated with the superscript $(n)$ represents the collection of the terms of $\mathcal{O}\left((\delta H / H)^{n}\right)$. On the right hand side of Eq. (4.12), all the terms except for the last one come from the initial zero mode. The direct expansion shows that the leading terms in Eqs. (4.12) and (4.13) exactly agree with each other up to the first order in $\delta H / H_{1}$,

$$
\begin{aligned}
\mathcal{P}_{5 \mathrm{D}}^{(0)}+\mathcal{P}_{5 \mathrm{D}}^{(1)}= & \mathcal{P}_{\text {res }}^{(0)}+\mathcal{P}_{\text {res }}^{(1)}=\frac{2 C^{2}\left(H_{1}\right)}{M_{\mathrm{Pl}}^{2}}\left(\frac{H_{1}}{2 \pi}\right)^{2} \\
& \times\left\{1+\left[\frac{\sin \left(2 p \eta_{0}\right)}{p \eta_{0}}-2\right] \frac{C^{2}\left(H_{1}\right)}{\sqrt{1+\left(\ell H_{1}\right)^{2}}} \frac{\delta H}{H_{1}}\right\} .
\end{aligned}
$$

These terms contain only the contribution from the initial zero mode.

The contribution from the initial $\mathrm{KK}$ modes $\mathcal{P}_{5 \mathrm{D}}^{\mathrm{KK}}$ is of order $\left(\delta H / H_{1}\right)^{2}$, because both $\alpha_{0 \kappa}$ and $\beta_{0 \kappa}$ are $\mathcal{O}\left(\delta H / H_{1}\right)$. Thus, to examine whether the agreement of the spectrum continues to hold even after including the KK modes, we investigate the second order part of the spectrum. The second order terms $\mathcal{P}_{5 \mathrm{D}}^{(2)}$ and $\mathcal{P}_{\text {res }}^{(2)}$ are given by 


$$
\begin{aligned}
\mathcal{P}_{5 \mathrm{D}}^{(2)}(p)= & \frac{2 C^{2}\left(H_{1}\right)}{M_{\mathrm{Pl}}^{2}}\left(\frac{H_{1}}{2 \pi}\right)^{2}\left[\left(p \eta_{0}\right)^{2}\left(\frac{C^{-2}\left(H_{1}\right)+C^{2}\left(H_{1}\right)}{\sqrt{1+\left(\ell H_{1}\right)^{2}}}-2\right)+\cos \left(p \eta_{0}\right)\left(\frac{C^{2}\left(H_{1}\right)}{\sqrt{1+\left(\ell H_{1}\right)^{2}}}+1\right)+\frac{\sin ^{2}\left(p \eta_{0}\right)}{\left(p \eta_{0}\right)^{2}} \frac{C^{2}\left(H_{1}\right)}{\sqrt{1+\left(\ell H_{1}\right)^{2}}}\right. \\
& \left.+\frac{\sin \left(2 p \eta_{0}\right)}{2 p \eta_{0}}\left(\frac{2+3\left(\ell H_{1}\right)^{2}}{1+\left(\ell H_{1}\right)^{2}}-\frac{6 C^{2}\left(H_{1}\right)}{\sqrt{1+\left(\ell H_{1}\right)^{2}}}\right)+\frac{4 C^{2}\left(H_{1}\right)}{\sqrt{1+\left(\ell H_{1}\right)^{2}}}-\frac{3+4\left(\ell H_{1}\right)^{2}}{1+\left(\ell H_{1}\right)^{2}}\right] \frac{C^{2}\left(H_{1}\right)}{\sqrt{1+\left(\ell H_{1}\right)^{2}}}\left(\frac{\delta H}{H_{1}}\right)^{2}, \\
\mathcal{P}_{\mathrm{res}}^{(2)}(p)= & \frac{2 C^{2}\left(H_{1}\right)}{M_{\mathrm{Pl}}^{2}}\left(\frac{H_{1}}{2 \pi}\right)^{2}\left[2 \cos \left(p \eta_{0}\right)+\frac{\sin ^{2}\left(p \eta_{0}\right)}{\left(p \eta_{0}\right)^{2}}+\frac{\sin \left(2 p \eta_{0}\right)}{p \eta_{0}}\left(\frac{2+3\left(\ell H_{1}\right)^{2}}{1+\left(\ell H_{1}\right)^{2}}-\frac{4 C^{2}\left(H_{1}\right)}{\sqrt{1+\left(\ell H_{1}\right)^{2}}}\right)\right. \\
& \left.-\frac{\sin ^{2}\left(2 p \eta_{0}\right)}{\left(2 p \eta_{0}\right)^{2}}\left(\frac{4+5\left(\ell H_{1}\right)^{2}}{1+\left(\ell H_{1}\right)^{2}}-\frac{4 C^{2}\left(H_{1}\right)}{\sqrt{1+\left(\ell H_{1}\right)^{2}}}\right)+\frac{4 C^{2}\left(H_{1}\right)}{\sqrt{1+\left(\ell H_{1}\right)^{2}}}-\frac{3+4\left(\ell H_{1}\right)^{2}}{1+\left(\ell H_{1}\right)^{2}}\right] \frac{C^{2}\left(H_{1}\right)}{\sqrt{1+\left(\ell H_{1}\right)^{2}}}\left(\frac{\delta H}{H_{1}}\right)^{2} .
\end{aligned}
$$

From these equations, we notice that $\mathcal{P}_{\text {res }}^{(2)}$ and $\mathcal{P}_{5 \mathrm{D}}^{(2)}$ do not agree with each other. We see that the difference is enhanced in particular at $p\left|\eta_{0}\right| \gg 1$. However, as we mentioned earlier, the KK mode contribution also gives a correction of the same order, and in fact we show that approximate agreement is recovered even in this order by adding the KK mode contribution.

First we observe the power spectrum at $p\left|\eta_{0}\right| \ll 1$. Expanding $\mathcal{P}_{5 \mathrm{D}}^{(2)}$ and $\mathcal{P}_{\text {res }}^{(2)}$ with respect to $p\left|\eta_{0}\right|$, we have

$$
\frac{\mathcal{P}_{5 \mathrm{D}}^{(2)}(p)}{\mathcal{P}^{(0)}}, \frac{\mathcal{P}_{\mathrm{res}}^{(2)}(p)}{\mathcal{P}^{(0)}} \sim\left(p \eta_{0}\right)^{2}\left(\frac{\delta H}{H_{1}}\right)^{2}
$$

On the other hand, Eq. (3.13) leads to

$$
\frac{\mathcal{P}_{5 \mathrm{D}}^{\mathrm{KK}}(p)}{\mathcal{P}^{(0)}} \sim\left(p\left|\eta_{0}\right|\right)^{3}\left(\frac{\delta H}{H_{1}}\right)^{2} .
$$

Therefore the difference is small outside the horizon as

$$
\left|\frac{\mathcal{P}_{5 \mathrm{D}}^{(2)}+\mathcal{P}_{5 \mathrm{D}}^{\mathrm{KK}}-\mathcal{P}_{\mathrm{res}}^{(2)}}{\mathcal{P}^{(0)}}\right| \sim\left(p \eta_{0}\right)^{2}\left(\frac{\delta H}{H_{1}}\right)^{2} \quad\left(p\left|\eta_{0}\right| \ll 1\right),
$$

although the cancellation between $\mathcal{P}_{5 \mathrm{D}}^{(2)}(p)$ and $\mathcal{P}_{\text {res }}^{(2)}(p)$ does not happen. By a similar argument, at $p\left|\eta_{0}\right| \lesssim 1$, we have

$$
\left|\frac{\mathcal{P}_{5 \mathrm{D}}^{(2)}+\mathcal{P}_{5 \mathrm{D}}^{\mathrm{KK}}-\mathcal{P}_{\mathrm{res}}^{(2)}}{\mathcal{P}^{(0)}}\right| \sim\left(\frac{\delta H}{H_{1}}\right)^{2} \quad\left(p\left|\eta_{0}\right| \lesssim 1\right)
$$

The situation is more interesting when we consider the spectrum inside the Hubble horizon. There is a term proportional to $\left(p \eta_{0}\right)^{2}$ in $\mathcal{P}_{5 \mathrm{D}}^{(2)}$, which is dominant at $p\left|\eta_{0}\right| \gg 1$, while there is no corresponding term in $\mathcal{P}_{\text {res }}^{(2)}$. Hence, the difference between $\mathcal{P}_{5 \mathrm{D}}^{(2)}$ and $\mathcal{P}_{\text {res }}^{(2)}$ is

$$
\begin{aligned}
\frac{\mathcal{P}_{\mathrm{5D}}^{(2)}-\mathcal{P}_{\mathrm{res}}^{(2)}}{\mathcal{P}^{(0)}} \approx & -\left(p \eta_{0}\right)^{2}\left(2-\frac{C^{-2}\left(H_{1}\right)+C^{2}\left(H_{1}\right)}{\sqrt{1+\left(\ell H_{1}\right)^{2}}}\right) \\
& \times \frac{C^{2}\left(H_{1}\right)}{\sqrt{1+\left(\ell H_{1}\right)^{2}}}\left(\frac{\delta H}{H_{1}}\right)^{2} .
\end{aligned}
$$

Our approximation is valid for $p\left|\eta_{0}\right| \delta H / H_{1} \lesssim 1$ [Eq. (2.27)]. Hence, within the region of validity, this difference can be as large as $\mathcal{P}^{(0)}$. We also note that the terms proportional to $\left(p\left|\eta_{0}\right|\right)^{2}$ come from $\alpha_{00}$, while the contribution from $\beta_{00}$ is suppressed at $p\left|\eta_{0}\right| \gg 1$. On the other hand, the contribution from initial KK modes, $\mathcal{P}_{5 \mathrm{D}}^{\mathrm{KK}}(p)$, is dominated by $\alpha_{0 \kappa}$ at $p\left|\eta_{0}\right| \gg 1: \mathcal{P}_{5 \mathrm{D}}^{\mathrm{KK}} \propto \int d \kappa\left|\alpha_{0 \kappa}+\beta_{0 \kappa}^{*}\right|^{2} \sim \int d \kappa\left|\alpha_{0 \kappa}\right|^{2}$. This means that, although the creation of zero-mode gravitons from the initial KK modes is negligible inside the horizon, a part of the amplitude of the final zero mode comes from the initial

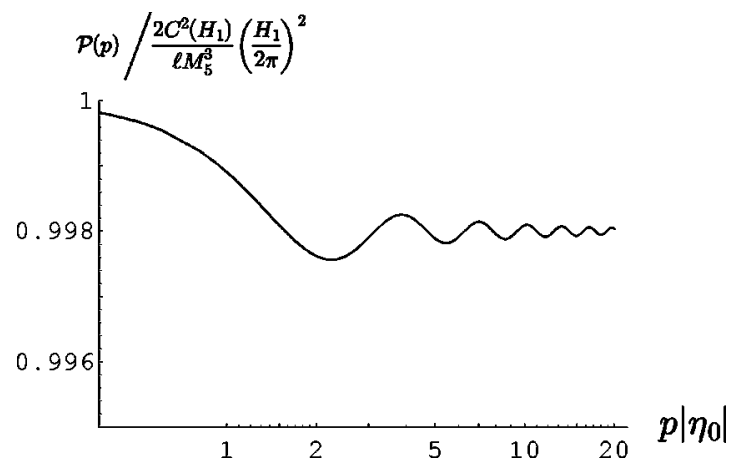

FIG. 3. Five-dimensional power spectrum of gravitational waves $\mathcal{P}_{5 \mathrm{D}}(p)$ and the four-dimensional one $\mathcal{P}_{4 \mathrm{D}}(p)$ at low energies $\left(\ell H_{1}=10^{-2}\right)$ with $\delta H / H_{1}=10^{-3}$. These two agree with each other. In this case the initial KK modes give a negligible contribution. 
KK modes losing their KK momenta. Since the coefficient $\alpha_{0 \kappa}$ is proportional to $p\left|\eta_{0}\right|$ at large $p\left|\eta_{0}\right|, \mathcal{P}_{5 \mathrm{D}}^{\mathrm{KK}}$ behaves as

$$
\frac{\mathcal{P}_{5 \mathrm{D}}^{\mathrm{KK}}(p)}{\mathcal{P}^{(0)}} \sim+\left(p \eta_{0}\right)^{2}\left(\frac{\delta H}{H_{1}}\right)^{2} .
$$

This KK mode contribution cancels $\mathcal{P}_{5}^{(2)} \mathrm{D}$. The cancellation can be proved by looking at the property of the Bogoliubov coefficients

$$
\left|\alpha_{00}\right|^{2}-\left|\beta_{00}\right|^{2}+\int d \kappa\left(\left|\alpha_{0 \kappa}\right|^{2}-\left|\beta_{0 \kappa}\right|^{2}\right)=1 .
$$

This relation together with Eq. (4.4) implies that the power spectrum cannot significantly deviate from $C^{2}\left(H_{2}\right) H_{2}^{2} / 2 \ell p^{3}$ in the region where $\beta_{00}$ and $\beta_{0 \kappa}$ are negligibly small. We can also demonstrate the cancellation by explicit calculation in the low and high energy limits. For $p\left|\eta_{0}\right| \gg 1$, we have $\alpha_{0 \kappa} \approx U_{0 \kappa}$. Then from Eqs. (2.20), (2.21), and (2.32) we obtain

$$
\int d \kappa\left|\alpha_{0 \kappa}\right|^{2} \approx\left\{\begin{array}{cc}
\int_{0}^{\infty} \frac{2 \kappa \tanh (\pi \kappa) d \kappa}{\left(\kappa^{2}+1 / 4\right)\left(\kappa^{2}+9 / 4\right)} \times\left(p \eta_{0}\right)^{2}\left(\ell H_{1}\right)^{2}\left(\frac{\delta H}{H_{1}}\right)^{2} & \left(\ell H_{1} \ll 1\right), \\
\frac{6}{\pi} \int_{0}^{\infty} \frac{\kappa^{2} d \kappa}{\left(\kappa^{2}+1 / 4\right)^{2}\left(\kappa^{2}+9 / 4\right)} \times\left(p \eta_{0}\right)^{2}\left(\frac{\delta H}{H_{1}}\right)^{2} & \left(\ell H_{1} \gg 1\right),
\end{array}\right.
$$

which gives $\left(p \eta_{0}\right)^{2}\left(\ell H_{1}\right)^{2}\left(\delta H / H_{1}\right)^{2}$ in the low energy regime and $(3 / 4)\left(p \eta_{0}\right)^{2}\left(\delta H / H_{1}\right)^{2}$ in the high energy regime. Comparing these with Eq. (4.21), we see that $\mathcal{P}_{5 \mathrm{DK}}^{\mathrm{KK}}(p)$ cancels $\mathcal{P}_{5 \mathrm{D}}^{(2)}(p)$. $^{2}$

To summarize, we have observed that the agreement between the rescaled spectrum $\mathcal{P}_{\text {res }}(p)$ and the fivedimensional spectrum $\mathcal{P}_{5 \mathrm{D}}(p)$ is exact up to first order in $\delta H / H_{1}$. The agreement is not exact at second order, but we found that the correction is not enhanced at any wavelength irrespective of the value of $\ell H_{1}$. Just for illustrative purpose we show the results of numerical calculations in Fig. 3 and Fig. 4.

\section{DISCUSSION}

In this paper we investigated the generation of primordial gravitational waves and its power spectrum in the inflationary braneworld model, focusing on the effects of the variation of the Hubble parameter during inflation. For this purpose, we considered a model in which the Hubble parameter changes discontinuously.

In the case of de Sitter inflation with constant Hubble parameter $H$, the spectrum is known to be given by Eq. (4.1) [13]. It agrees with the standard four-dimensional one [Eq. (4.2)] at low energies $\ell H \ll 1$, but at high energies $\ell H \gg 1$ it significantly deviates from Eq. (4.2) due to the amplification effect of the zero-mode normalization factor $C(H)$. One can say, however, that the five-dimensional spectrum is obtained from the four-dimensional one by the map $H \mapsto H C(H)$.

In a model with variable Hubble parameter, gravitational wave perturbations are expected to be generated not only from the "in vacuum" of the zero mode but also from that of the Kaluza-Klein modes. Hence, it is not clear whether there

\footnotetext{
${ }^{2}$ The small $\ell H$ expansion $C^{2}(H) \approx 1-(\ell H)^{2}[1 / 2+\ln (\ell H / 2)]$ is used here to investigate the low energy case.
}

is a simple relation between the five-dimensional spectrum and the four-dimensional counterpart. Analyzing the model with a discontinuous jump in the Hubble parameter, we have shown that this is indeed approximately the case. More precisely, if the squared amplitude of four-dimensional fluctuations is given by $\left(h / 2 \pi M_{\mathrm{Pl}}\right)^{2}$, we transform $\left(h / 2 \pi M_{\mathrm{Pl}}\right)^{2}$ into $C^{2}(h)\left(h / 2 \pi M_{\mathrm{Pl}}\right)^{2}$, then the resulting rescaled spectrum exactly agrees with the five-dimensional spectrum $\mathcal{P}_{5 \mathrm{D}}(p)$ up to first order in $\delta H / H_{1}$. At second order $\mathcal{O}(\delta H / H)^{2}$ the agreement is not exact, but the difference is not enhanced at any wavelength irrespective of the value of $\ell H_{1}$. Hence, in total, the agreement is not significantly disturbed by the mismatch at second order. As a nontrivial point, we also found that the initial KK mode vacuum fluctuations can give a non-negligible contribution to the final zero-mode states at second order.

One may expect that the power spectrum of gravitational waves in the braneworld model would reflect the character-

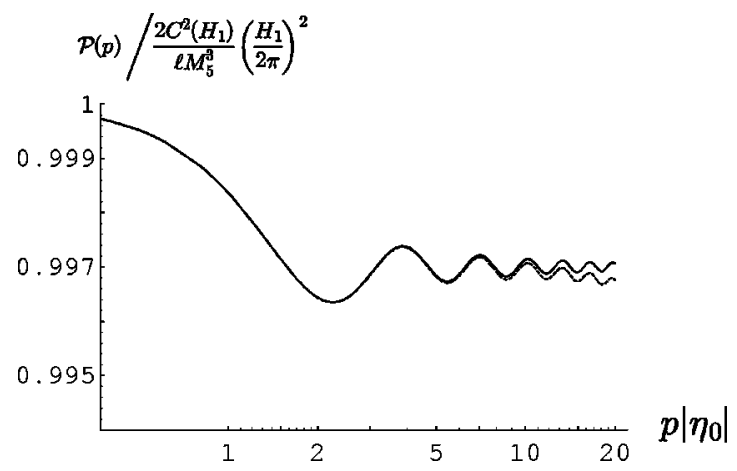

FIG. 4. Power spectra of gravitational waves at high energies $\left(\ell H_{1}=10^{3}\right)$ with $\delta H / H_{1}=10^{-3}$. Five-dimensional spectrum $\mathcal{P}_{5 \mathrm{D}}^{\text {zero }}(p)+\mathcal{P}_{5 \mathrm{D}}^{\mathrm{KK}}(p)$ and the rescaled four-dimensional one $\mathcal{P}_{\text {res }}(p)$ agree well with each other (solid lines), while only the zero-mode contribution $\mathcal{P}_{5 \mathrm{D}}^{\text {zero }}(p)$ gives a reduced fluctuation amplitude inside the horizon (dotted line). 
istic length scale corresponding to the curvature (or "compactification") scale of the extra dimension $\ell$. However, our analysis showed that the resultant power spectrum does not depend on the ratio of the wavelength of gravitational waves to the bulk curvature scale, $p\left|\eta_{0}\right| \ell H$.

Here we should mention the result of Ref. [18] that is summarized in Appendix A. Their setup is the most violent version of the transition $H_{1} \rightarrow H_{2}=0$. If the wavelength of the gravitational waves is much longer than both the Hubble scale and the bulk curvature scale, the power spectrum is given by Eq. (A4) and obviously it is obtained from the four-dimensional counterpart by the map $H \mapsto H C(H)$. However, if the wavelength is longer than the Hubble scale but much smaller than the bulk curvature radius, the amplitude is highly damped as is seen from Eq. (A7) and the map $H \mapsto H C(H)$ does not work at all. This damping of the amplitude can be understood in the following way. In the high energy regime $\ell H \gg 1$, the motion of the brane with respect to the static bulk is ultrarelativistic. At the moment of transition to the Minkowski phase, the brane abruptly stops. Zero-mode gravitons with wavelength smaller than the bulk curvature scale can be interpreted as "particles" traveling in five dimensions. These gravitons make a "hard hit" with the brane at the moment of this transition, and get large momenta in the fifth direction relative to the static brane. As a result, these gravitons escape into the bulk as KK gravitons, and thus the amplitude (A7) is damped. On the other hand, in our model the change of the Hubble parameter is assumed to be small, and hence such violent emission of KK gravitons does not happen. If this interpretation is correct, the mapping rule $h \mapsto h C(h)$ will generally give a rather good estimate for the prediction of inflationary braneworld models as long as the time variation of the Hubble parameter is smooth. If we can confirm the validity of this prescription in more general cases, the analysis of gravitational wave perturbations will be simplified a lot. We would like to return to this issue of generalization in a future publication.

\section{ACKNOWLEDGMENTS}

H.K. is supported by the JSPS. To complete this work, the discussion during and after the YITP workshop YITP-W02-19 was useful. This work is partly supported by the Monbukagakusho Grant-in-Aid No. 1470165 (T.T.).

\section{APPENDIX A: PARTICLE CREATION WHEN CONNECTED TO MINKOWSKI BRANE}

Here for comparison with our results we briefly summarize the results obtained by Gorbunov et al. [18] focusing on the power spectrum of gravitational waves. Their method is basically the same as that we already explained in the main text. They considered the situation that de Sitter inflation on the brane with constant Hubble parameter $H$ suddenly terminates at a conformal time $\eta=\eta_{0}$, and is followed by a Minkowski phase. The power spectrum of gravitational waves is expressed in terms of the Bogoliubov coefficients as

$$
\mathcal{P}_{5 \mathrm{D}}(p)=\frac{2}{M_{\mathrm{Pl}}^{2}}\left(\frac{H}{2 \pi}\right)^{2}\left(p \eta_{0}\right)^{2}\left(1+2\left|\beta_{00}\right|^{2}+2 \int d \kappa\left|\beta_{0 \kappa}\right|^{2}\right),
$$

where $\left|\beta_{00}\right|^{2}$ and $\left|\beta_{0 \kappa}\right|^{2}$ are the number of zero-mode gravitons created from initial zero-mode and KK modes, respectively. At super Hubble scale $\left(p\left|\eta_{0}\right| \ll 1\right)$ we can neglect the first term in the parentheses, which corresponds to the vacuum fluctuations in Minkowski space.

According to [18], when $p\left|\eta_{0}\right| \ell H \ll 1$ [i.e., the wavelength of the gravitational wave $(p / a)^{-1}$ is much larger than the bulk curvature scale $\ell]$ and $p\left|\eta_{0}\right| \ll 1$, the coefficients are given by

$$
\begin{gathered}
\left|\beta_{00}\right|^{2} \approx \frac{C^{2}(H)}{4\left(p \eta_{0}\right)^{2}}, \\
\int d \kappa\left|\beta_{0 \kappa}\right|^{2} \sim\left\{\begin{array}{cc}
p\left|\eta_{0}\right|(\ell H)^{2} & (\ell H \ll 1), \\
p\left|\eta_{0}\right| \ell H & (\ell H \gg 1) .
\end{array}\right.
\end{gathered}
$$

One can see that the contribution from initial KK modes is suppressed irrespective of the expansion rate $\ell H$. Therefore the power spectrum is evaluated as

$$
\mathcal{P}_{5 \mathrm{D}} \approx \frac{C^{2}(H)}{M_{\mathrm{Pl}}^{2}}\left(\frac{H}{2 \pi}\right)^{2} .
$$

Equation (A4) is half of the power spectrum on the de Sitter brane [Eq. (4.1)], and this result can be understood as follows. The amplitude of fluctuations at the super Hubble scale stays constant. After the sudden transition from the de Sitter phase to the Minkowski phase, the Hubble scale becomes infinite. Therefore, those fluctuation modes are now inside the Hubble horizon, and they begin to oscillate. As a result, the mean-square vacuum fluctuation becomes half of the initial value.

On the other hand, when $p\left|\eta_{0}\right| \ell H \gg 1$ and $p\left|\eta_{0}\right| \ll 1$ (these conditions require $\ell H \gg 1$ ), the Bogoliubov coefficients are given by

$$
\begin{aligned}
\left|\beta_{00}\right|^{2} & \approx \frac{C^{2}(H)}{\left(p \eta_{0}\right)^{2}} \frac{1}{\left(p \eta_{0} \ell H\right)^{2}}, \\
\int d \kappa\left|\beta_{0 \kappa}\right|^{2} & \sim \frac{1}{\left(p \eta_{0} \ell H\right)^{2}} .
\end{aligned}
$$

As before, the contribution from the initial KK modes is negligible and that from $\left|\beta_{00}\right|^{2}$ dominates the power spectrum,

$$
\mathcal{P}_{5 \mathrm{D}} \approx \frac{C^{2}(H)}{M_{\mathrm{Pl}}^{2}}\left(\frac{H}{2 \pi}\right)^{2} \frac{4}{\left(p \eta_{0} \ell H\right)^{2}} .
$$

One can see that the spectrum is suppressed by the factor $4 /\left(p \eta_{0} \ell H\right)^{2}$. 


\section{APPENDIX B: CALCULATIONS \\ OF THE INNER PRODUCT}

which reduce to the following forms at the infinite past ( $\eta$ $\rightarrow-\infty)$ :

We derive Eqs. (2.31) and (2.32) by calculating the inner product (2.30). Expanding Eq. (2.25) in terms of $\epsilon_{H}$, we have

$$
\tilde{\eta}-\eta=\epsilon_{H} \eta_{0} \cosh \xi
$$

$$
\begin{aligned}
& \tilde{\eta}=\eta+\epsilon_{H} \eta_{0} \cosh \xi-\epsilon_{H}^{2} \eta_{0}^{2}(\sinh \xi)^{2} /(2 \eta)+\cdots, \\
& \tilde{\xi}=\xi-\epsilon_{H} \eta_{0} \sinh \xi / \eta+\epsilon_{H}^{2} \eta_{0}^{2} \cosh \xi \sinh \xi / \eta^{2}+\cdots,
\end{aligned}
$$

$$
\widetilde{\xi}-\xi=0 .
$$

(B2) Then, $U_{00}$ is evaluated at $\eta=-\infty$ as

$$
\begin{aligned}
& U_{00}=-\left.2 i \ell^{3} \int_{\xi_{B}}^{\infty} \frac{d \xi}{\eta^{2}(\sinh \xi)^{3}}\left(\widetilde{\phi}_{0}^{(-)} \partial_{\eta} \phi_{0}^{(+)}-\phi_{0}^{(+)} \partial_{\eta} \widetilde{\phi}_{0}^{(-)}\right)\right|_{\eta \rightarrow-\infty}=\frac{H_{2}}{H_{1}} C\left(H_{1}\right) C\left(H_{2}\right) \cdot 2 \ell^{2} H_{1}^{2} \int_{\xi_{B}}^{\infty} d \xi \frac{e^{-i \epsilon_{H} p \eta_{0} \cosh \xi}}{(\sinh \xi)^{3}}
\end{aligned}
$$

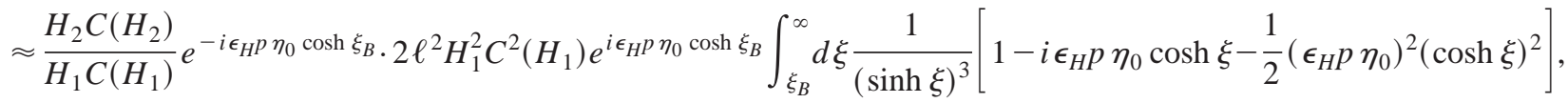

where we expanded the integrand with respect to $\epsilon_{H}$ in the last line. Integrating each term, we finally obtain

$$
U_{00} \approx \frac{H_{2} C\left(H_{2}\right)}{H_{1} C\left(H_{1}\right)} e^{-i \epsilon_{H} p \eta_{0} \cosh \xi_{B}}\left[1-i \epsilon_{H} p \eta_{0}\left(C^{2}\left(H_{1}\right)-\cosh \xi_{B}\right)-\frac{1}{2}\left(\epsilon_{H} p \eta_{0}\right)^{2}\left(\sinh \xi_{B}\right)^{2}\right] .
$$

Note that the condition $\epsilon_{H} p\left|\eta_{0}\right| \cosh \xi_{B}\left(\approx p\left|\eta_{0}\right| \delta H / H\right) \ll 1$ is required in order to justify the expansion of the exponent. Because the integral is saturated at $\xi \approx \xi_{B}$, we do not have to worry about the validity of the expansion for large $\cosh \xi$.

The explicit form of $U_{0 \kappa}$ is needed up to the first order in $\epsilon_{H}$. A similar calculation leads to

$$
\begin{aligned}
U_{0 \kappa}= & -\left.2 i \ell^{3} \int_{\xi_{B}}^{\infty} \frac{d \xi}{\eta^{2}(\sinh \xi)^{3}}\left(\widetilde{\phi}_{0}^{(-)} \partial_{\eta} \phi_{\kappa}^{(+)}-\phi_{\kappa}^{(+)} \partial_{\eta} \widetilde{\phi}_{0}^{(-)}\right)\right|_{\eta \rightarrow-\infty}=-2 i \ell^{3} \cdot \ell^{-1 / 2} C\left(H_{2}\right) \frac{H_{2}}{\sqrt{2 p}} \cdot \frac{\ell^{-3 / 2}}{\sqrt{2 p}} \int_{\xi_{B}}^{\infty} \frac{d \xi}{\eta^{2}(\sinh \xi)^{3}} \chi_{\kappa}(\xi) \\
& \times\left.\left[\left(\tilde{\eta}-\frac{i}{p}\right) e^{-i p \tilde{\eta}} \cdot(-1-i p \eta) e^{i p \eta+i \pi / 4}-(-\tilde{\eta}) e^{i p \eta+i \pi / 4} \cdot(-i p \eta) e^{-i p \tilde{\eta}}\right]\right|_{\eta \rightarrow-\infty} \\
= & -2 e^{i \pi / 4} \ell H_{2} C\left(H_{2}\right) \int_{\xi_{B}}^{\infty} d \xi \frac{e^{-i \epsilon_{H} p \eta_{0} \cosh \xi_{B}}}{(\sinh \xi)^{3}} \chi_{\kappa}(\xi) .
\end{aligned}
$$

The integral in the last line, which we call $I$, can be calculated as follows. Again, expanding the integrand in terms of $\epsilon_{H}$, we have

$$
I \approx \int_{\xi_{B}}^{\infty} d \xi \frac{\chi_{\kappa}(\xi)}{(\sinh \xi)^{3}}\left(1-i \epsilon_{H} p \eta_{0} \cosh \xi\right)
$$

Let us consider the first term in the parentheses. The spatial wave function $\chi_{\kappa}$ satisfies $(\sinh \xi)^{-3} \chi_{\kappa}=-\left(\kappa^{2}\right.$ $+9 / 4)^{-1} \partial_{\xi}\left[(\sinh \xi)^{-3} \partial_{\xi} \chi_{\kappa}\right]$ [Eq. (2.10)] with the boundary condition $\partial_{\xi} \chi_{\kappa}\left(\xi_{B}\right)=0$. Therefore, together with the behavior at infinity, $(\sinh \xi)^{-3} \partial_{\xi} \chi_{\kappa} \sim(\sinh \xi)^{-3} \partial_{\xi}(\sinh \xi)^{3 / 2} \rightarrow 0$, we find that the integral of the first term vanishes. Then, using integration by parts twice, we have 


$$
\begin{aligned}
\left(\kappa^{2}+\frac{9}{4}\right) I & \approx i \epsilon_{H} p \eta_{0} \int_{\xi_{B}}^{\infty} d \xi \cosh \xi \frac{\partial}{\partial \xi}\left[\frac{1}{(\sinh \xi)^{3}} \frac{\partial}{\partial \xi} \chi_{\kappa}(\xi)\right] \\
& =-i \epsilon_{H} p \eta_{0} \int_{\xi_{B}}^{\infty} d \xi \frac{1}{(\sinh \xi)^{2}} \frac{\partial}{\partial \xi} \chi_{\kappa}(\xi) \\
& =i \epsilon_{H} p \eta_{0} \frac{\chi_{\kappa}\left(\xi_{B}\right)}{\left(\sinh \xi_{B}\right)^{2}}+i \epsilon_{H} p \eta_{0} \int_{\xi_{B}}^{\infty} d \xi \frac{-2 \cosh \xi}{(\sinh \xi)^{3}} \chi_{\kappa}(\xi) \\
& \approx i \epsilon_{H} p \eta_{0} \frac{\chi_{\kappa}\left(\xi_{B}\right)}{\left(\ell H_{1}\right)^{2}}+2 I,
\end{aligned}
$$

from which we can evaluate $U_{0 \kappa}$. Note that the approximation is valid when $p\left|\eta_{0}\right| \delta H / H_{1} \ll 1$.

[1] N. Arkani-Hamed, S. Dimopoulos, and G.R. Dvali, Phys. Lett. B 429, 263 (1998); Phys. Rev. D 59, 086004 (1999).

[2] L. Randall and R. Sundrum, Phys. Rev. Lett. 83, 3370 (1999).

[3] L. Randall and R. Sundrum, Phys. Rev. Lett. 83, 4690 (1999).

[4] J. Garriga and T. Tanaka, Phys. Rev. Lett. 84, 2778 (2000).

[5] H. Kudoh and T. Tanaka, Phys. Rev. D 64, 084022 (2001); I. Giannakis and H.C. Ren, ibid. 63, 024001 (2001); T. Wiseman, ibid. 65, 124007 (2002).

[6] D. Langlois, Prog. Theor. Phys. Suppl. 148, 181 (2003).

[7] P. Binetruy, C. Deffayet, U. Ellwanger, and D. Langlois, Phys. Lett. B 477, 285 (2000); P. Kraus, J. High Energy Phys. 12, 011 (1999); S. Mukohyama, Phys. Lett. B 473, 241 (2000); D. Ida, J. High Energy Phys. 09, 014 (2000).

[8] J. Garriga and M. Sasaki, Phys. Rev. D 62, 043523 (2000).

[9] T. Nihei, Phys. Lett. B 465, 81 (1999); N. Kaloper, Phys. Rev. D 60, 123506 (1999); H.B. Kim and H.D. Kim, ibid. 61, 064003 (2000).

[10] Y. Himemoto and M. Sasaki, Phys. Rev. D 63, 044015 (2001); Y. Himemoto, T. Tanaka, and M. Sasaki, ibid. 65, 104020 (2002).

[11] S. Kobayashi, K. Koyama, and J. Soda, Phys. Lett. B 501, 157 (2001).
[12] R. Maartens, D. Wands, B.A. Bassett, and I.P.C. Heard, Phys. Rev. D 62, 041301(R) (2000).

[13] D. Langlois, R. Maartens, and D. Wands, Phys. Lett. B 489, 259 (2000).

[14] V. Sahni, M. Sami, and T. Souradeep, Phys. Rev. D 65, 023518 (2002).

[15] N. Deruelle, Astrophys. Space Sci. 283, 619 (2003).

[16] H. Kodama, A. Ishibashi, and O. Seto, Phys. Rev. D 62, 064022 (2000); D. Langlois, ibid. 62, 126012 (2000); Phys. Rev. Lett. 86, 2212 (2001).

[17] K. Koyama and J. Soda, Phys. Rev. D 62, 123502 (2000); K. Koyama and J. Soda, ibid. 65, 023514 (2002).

[18] D.S. Gorbunov, V.A. Rubakov, and S.M. Sibiryakov, J. High Energy Phys. 10, 015 (2001).

[19] A.V. Frolov and L. Kofman, "Gravitational Waves from Braneworld Inflation," hep-th/0209133.

[20] K. Koyama and K. Takahashi, Phys. Rev. D 67, 103503 (2003).

[21] G.F. Giudice, E.W. Kolb, J. Lesgourgues, and A. Riotto, Phys. Rev. D 66, 083512 (2002).

[22] B. Allen and S. Koranda, Phys. Rev. D 50, 3713 (1994). 